\title{
Density Distribution Measurements in Rarefied Gases Contained between Parallel Plates at High Temperature Differences
}

\author{
Darryl J. Alofs, Richard C. Flagan, and George S. Springer \\ Fluid Dynamics Laboratory, Department of Mechanical Engineering \\ The University of Michigan, Ann Arbor, Michigan 48104
}

(Received 5 June 1970)

\begin{abstract}
Density distributions were experimentally determined in rarefied gases at rest contained between two parallel plates maintained at widely different temperatures. The temperatures of the hot and cold plates were $\sim 79^{\circ} \mathrm{K}$ and $\sim 294^{\circ} \mathrm{K}$, respectively, resulting in a temperature ratio of about $4: 1$. Both helium and nitrogen were used as test gases. Particular attention was focused on obtaining data in the temperature jump and transition regimes. The gas densities were measured by observing the luminescence produced by a high-energy electron beam traversed between the plates. In addition to the density measurements, heat transfer measurements were also made in order to estimate the values of the thermal accommodation coefficients. The experimental results for helium were compared with the analytical results of Liu and Lees, and good agreement was found between the theory and data near the cold plate. Near the hot plate the analytical results and the data differed by about $7 \%$.
\end{abstract}

\section{INTRODUCTION}

Experimental data for heat transfer and density distributions through a rarefied gas contained between parallel plates having different temperatures $\left(T_{H}\right.$ and $T_{c}$ ) are available only for the case of small temperature differences between the plates $\left(1-T_{C} / T_{H}<1\right){ }^{1}$ The objective of this investigation was to measure local gas densities in rarefied gases at rest contained between parallel plates maintained at high temperature differences $\left(T_{H} / T_{C}\right.$ $\approx 4 / 1$ ). Attention was focused on the temperature jump and transition regimes, the measurements having been performed in the Knudsen number range $\mathrm{Kn} \approx 0.03-0.6$. The Knudsen number is here defined as the ratio of the mean free path $\lambda_{0}$ at the center plane (i.e., at $x=0$, Fig. 1 ) to the distance between the plates, $L$.

The local gas densities were determined by measuring the luminescence produced by the passage of a narrow beam of high-energy electrons through the test gas. The beam was directed between the plates and parallel to them (Fig. 1). Gas densities at different positions between the plates were measured by moving the plates relative to the beam. In addition to the density measurements, heat transfer measurements were also made in the free molecule regime in order to evaluate the average values of the thermal accommodation coefficients at the hot and the cold surfaces.

\section{EXPERIMENTAL APPARATUS}

The experimental apparatus, shown schematically in Figs. 1 and 2, consisted of (1) a flat plate assembly, (2) an electron gun system for generating the electron beam, (3) two optical systems for

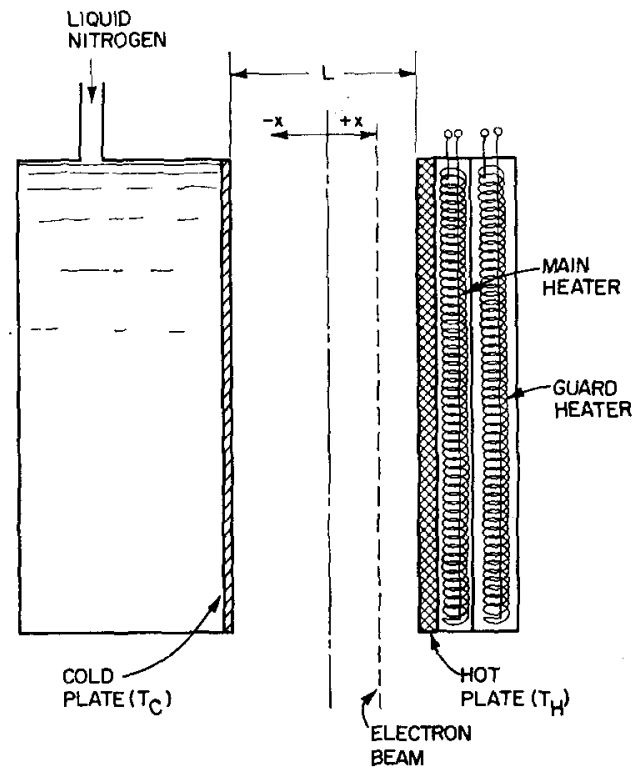

Fra. 1. Schematic of the experiment.

measuring the luminescence from the desired sections of the beam, and (4) a vacuum system.

The plate assembly (Fig. 1) consisted of an electrically heated (hot) plate and a liquid nitrogen cooled (cold) plate. Both the hot and cold plates were square $(20.32 \mathrm{~cm} \times 20.32 \mathrm{~cm})$, and were mounted in a vertical position on a movable platform (Fig. 2). The position of this platform (and therefore the position of the plates relative to the electron beam) could be adjusted to within 0.0025 $\mathrm{cm}$ by a screw mechanism. The distance $L$ between the plates was kept constant during the experiments. During the heat transfer measurements there was only a small distance $(L=0.0735 \mathrm{~cm})$ between the hot and cold plates. For the density 


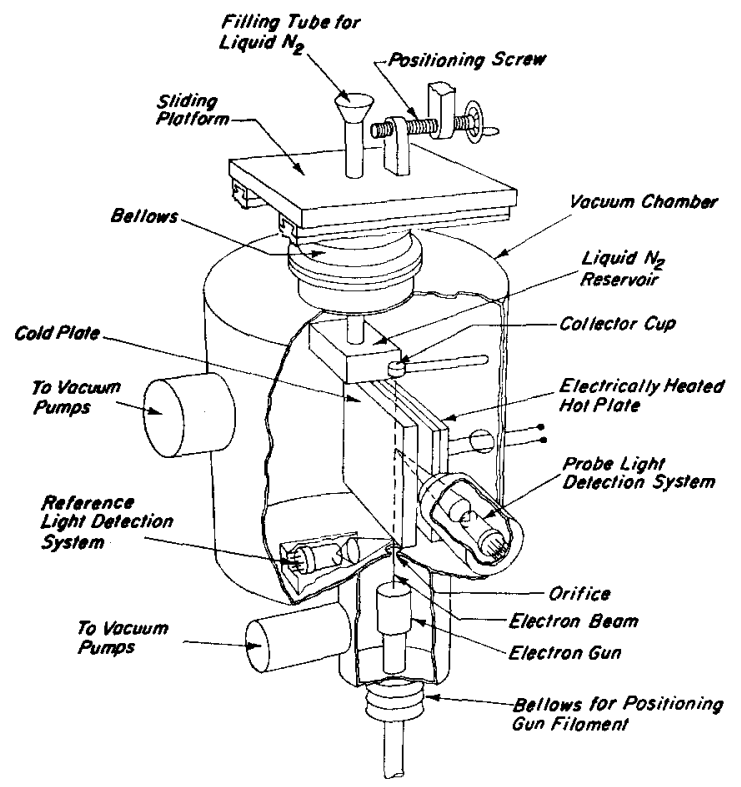

FIG. 2. Schematic of experimental apparatus.

distribution measurements this distance was increased to $2.28 \mathrm{~cm}$ by a suitable mechanism without disturbing the vacuum.

The cold plate was made of stainless steel, and the hot plate of aluminum. The hot plate was heated electrically as shown in Fig. 1. In addition to the main heater the hot plate was also equipped with a guard heater to minimize end losses from the back side of the plate. The power inputs to the main and guard heaters were determined by measuring the potential drop across the wires leading directly into the heating elements, and across a standard $1 \Omega$ resistance placed in series in the circuit. The temperatures of the hot and the cold plates $\left(T_{H}\right.$ and $\left.T_{C}\right)$ were monitored with eight thermocouples. The temperatures $T_{H}$ and $T_{C}$ were maintained at $79 \pm 0.5^{\circ} \mathrm{K}$ and $294 \pm 1^{\circ} \mathrm{K}$ during the experiments.

The electron beam system consisted of an electron gun capable of producing a narrow, high-energy electron beam and a collector cup which was used to measure the electron beam current. The electron gun was placed in a vacuum chamber in which the pressure could be maintained below $1 \times 10^{-4} \mathrm{~mm} \mathrm{Hg}$ throughout the experiments. There was a small $(0.2 \mathrm{~cm}$ diam) hole between the test chamber and the vacuum chamber housing the gun. The electron gun was the type commonly used in television tubes, but the commercial cathode was replaced with a tungsten filament. The electron beam was collected in a shielded cup and the electron beam current (in the range of 1-2 $\mu \mathrm{A}$ ) was measured with a microammeter. Two sets of electrostatic deflection plates were also provided to adjust the beam position, so that the beam passed through the hole between the gun chamber and test chamber, and that it also entered the $0.1 \mathrm{~cm}$ hole in the collector cup. These two holes were aligned paraliel to the plates, and thus the electron beam was also parallel to the plates. The position of the beam was constant during the experiments, and the plates were moved relative to the beam.

Two light detection systems were used in making the density measurements, each system measuring light intensity from a different length section of the beam. For the main detector, this length section was $1 \mathrm{~cm}$ long located midway between the top and bottom of the plates (Fig. 2). For the reference detector this length section was $3 \mathrm{~cm}$ long, and was located in the test chamber about $12 \mathrm{~cm}$ below the plates (Fig. 2). The output of the main detector was used for determining the density between the plates. The purpose of the reference detector was to record any small fluctuations $( \pm 2 \%)$ in the chamber pressure. This information was then used to correct the final data for these pressure fluctuations. Each detector had a lens system which focused an image of the luminescent beam on a slit which served as an optical stop. Directly behind the slit was a model $6655 \mathrm{~A}$ RCA photomultiplier tube, whose output was measured with an electrometer and recorded on a strip chart recorder.

The vacuum system (Fig. 2) was constructed of type 304 stainless steel. The test chamber (45 $\mathrm{cm}$ diam, $45 \mathrm{~cm}$ high) and the gun chamber (12.7 cm diam, $25 \mathrm{~cm}$ high) were equipped with separate diffusion and mechanical pumps. On the side of the test chamber was a 6.35 -cm-diam window for the optical system. The lowest pressure attainable in the test section was about $1 \times 10^{-6} \mathrm{~mm} \mathrm{Hg}$. The test gases (commercially pure helium and nitrogen) were admitted into the test chamber through adjustable leaks. The test chamber was equipped with an ionization gauge, and two precision McLeod gauges. The former could be read continuously, and therefore this was used for checking that the chamber pressure remained at a constant value during the experiments. However, the actual value of the chamber pressure was always measured with the McLeod gauges. No part of the apparatus was baked during the experiments.

\section{EXPERIMENTAL PROCEDURES}

During the density measurements the vacuum chamber pressure $P$ and the temperatures $T_{H}$ and $T_{c}$ were kept constant and the luminescence pro- 
duced by the passage of the electron beam through the gas was measured. Under the conditions of the present experiments and for constant beam energy the intensity of luminescence $I(x)$ is proportional to the local density $\rho(x)$, i.e.,

$$
I(x)=K(x) \rho(x),
$$

where the distance $x$ is measured from the plate centerplane, $x$ being positive toward the hot plate (Fig. 1). $K(x)$ is a constant that depends only on the beam position $x$. The reason for this dependence is that the optical system allowed some stray light to reach the photomultiplier tube. $K(x)$ was determined by setting $T_{H}=T_{C}$ (both being equal to the room temperature) and by measuring $I(x)$ at different but known values of $P$. Knowing $P$ and $T$, the density $\rho$ is found from the ideal gas law, and $K$ is evaluated from Eq. (1). The closest distance between the beam and the walls was $0.5 \mathrm{~cm}$. When the beam was closer to the walls, experimental accuracy decreased because wall fluorescence became too strong.

In addition to the density measurements heat transfer measurements between parallel plates were also made to evaluate the thermal accommodation coefficients at the hot and cold surfaces $\left(\alpha_{H}\right.$ and $\left.\alpha_{C}\right)$. For large temperature differences $\left(T_{c} \approx 79^{\circ} \mathrm{K}, T_{H} \approx 294^{\circ} \mathrm{K}\right.$ ) these measurements were made with the system described above, and for small temperature differences $\left(T_{H} \approx T_{C} \approx 294^{\circ} \mathrm{K}\right)$ with a plane layer type thermal conductivity cell described in detail in Refs. 1 and 2. The material and surface finish of the plates used in the latter apparatus were the same as that of the hot plate shown in Fig. 1. All the heat transfer measurements were performed at low pressures where Knudsen's formula ${ }^{3}$ approximates the heat conducted between the plates (free molecule conditions, $\mathrm{Kn}=\lambda / L>$ 10) quite well.

When the temperature difference between the plates is small (both plate temperatures being close to room temperature), the density between the plates may be closely approximated by the known density in the vacuum chamber. In this case, the thermal accommodation coefficient can be evaluated in a straight forward manner as described in Refs. 1 and 2 . The results of these measurements yield $\alpha_{H}$ which were found to be 0.58 and 0.82 for helium and nitrogen, respectively. To determine $\alpha_{C}$ heat transfer measurements were also made at large temperature differences. Now, however, the gas density between the plates is different from the density in the chamber and, therefore, $\alpha_{C}$ cannot be evaluated precisely. From the data $\alpha_{C}$ were estimated to be in the range $0.4-0.58$ for helium and 0.7-0.82 for nitrogen. Lacking further information on $\alpha_{C}$, in the following comparisons between theory and data, we shall use $\alpha_{C}=\alpha_{H}$.

\section{RESULTS}

Experimental data for helium and nitrogen are presented in Figs. 3 and 4 . The mean free paths used in calculating the Knudsen numbers $\left(\mathrm{Kn}_{0}=\right.$ $\left.\lambda_{0} / L\right)$ were based on the density measured at the center plane $\left(\rho=\rho_{0}\right.$ at $\left.\bar{x}=x / L=0\right)$, and on the assumption that the gas is composed of hard sphere molecules. Of course, the local Knudsen numbers are higher than $\mathrm{Kn}_{0}$ toward the hot plate and lower than $\mathrm{Kn}_{0}$ toward the cold plate.

An error analysis of the data has been made indicating that the systematic errors in the experiments were less than about $\pm 2 \%$. This error analysis indicated that the maximum error would be near the cold plate. The good agreement between the results of the continuum analysis $\left(\mathrm{Kn}_{0} \rightarrow 0\right)$ and the data near the cold plate at low Knudsen numbers $\left(\mathrm{Kn}_{0}=0.033\right)$ suggests that the error in the
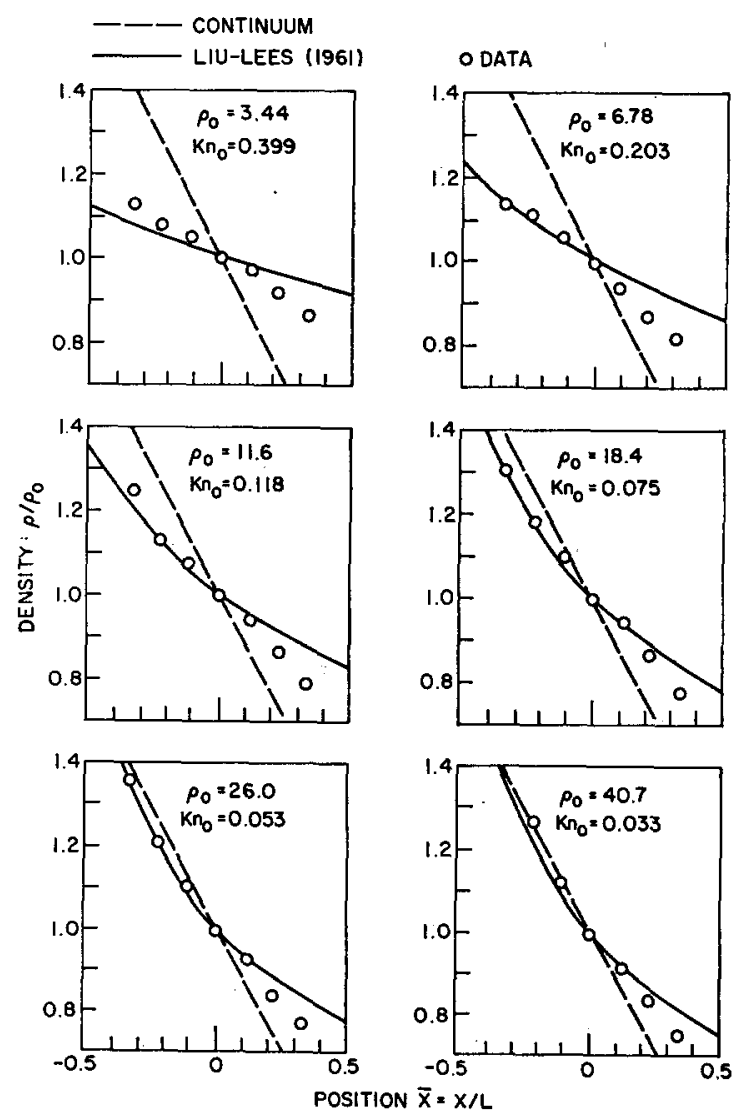

FIG. 3. Density ratio versus position. (Helium, $T_{H}=294^{\circ} \mathrm{K}$, $T_{C}=79^{\circ} \mathrm{K}, \alpha_{H}=\alpha_{C}=0.58, \mathrm{Kn}_{0} \equiv \lambda_{0} / L, \rho_{0}=10^{-9} \mathrm{~g} / \mathrm{cm}^{3}$.) 


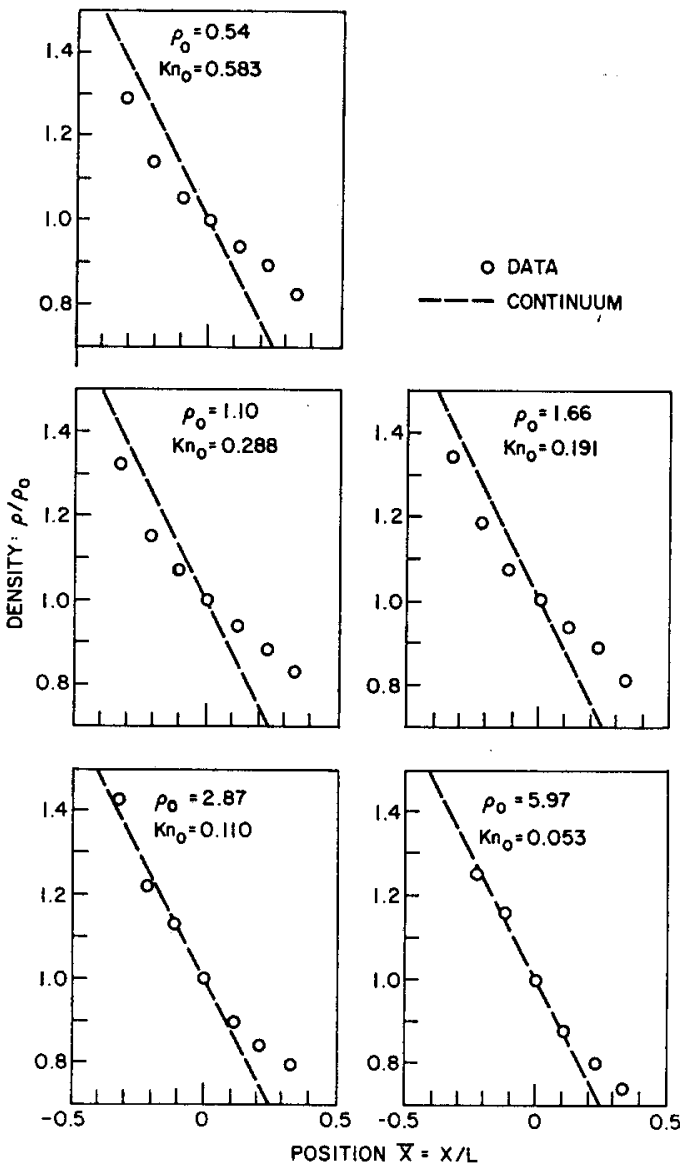

FIg. 4. Density ratio versus position. (Nitrogen, $T_{H}=294^{\circ} \mathrm{K}^{\prime}$ $T_{C}=79^{\circ} \mathrm{K}, \alpha_{H}=\alpha_{C}=0.82, \mathrm{Kn}_{0} \equiv \lambda_{0} / L, \rho_{0}=10^{-9} \mathrm{~g} / \mathrm{cm}^{3}$.)

measured density values was less than this estimated maximum. Possible errors may also be introduced into the density measurements by natural convection effects and by end effects. The natural convection effects would be most significant at low Knudsen numbers. However, even at the lowest Knudsen numbers the gas density was of the order of $10^{-8} \mathrm{~g} / \mathrm{cm}^{3}$. Previous experimental evidence ${ }^{4,5}$ indicates that under such conditions convection effects are negligible. The good agreement between the data and the results of the continuum analysis near the cold plate at low Knudsen numbers bears out this result. End effects would influence the data most near the hot plate, where the mean free path is the longest. A discussion of these effects will be given later.

The experimentally determined density distributions may be compared to the results of various theoretical methods proposed for describing heat conduction and density distributions in rarefied gases contained between parallel plates maintained at high temperature differences. For the present ranges of experimental conditions (i.e., $T_{H} / T_{C}>1$ and $0<\mathrm{Kn}<1$ ) analytical solutions were obtained for monatomic gases by Cercignani and Tironi, ${ }^{6}$ Willis, ${ }^{7}$ Liu and Lees, ${ }^{8}$ and Lavin and Haviland. ${ }^{9}$ Cercignani and Tironi, and Willis analyzed the problem by applying a numerical integration method of solution to the BGK model equation, while Liu and Lees, and Lavin and Haviland utilized a full range moment method solution developed by Lees. ${ }^{10}$ Liu and Lees obtained solutions using four moments and assuming Maxwellian molecules; I avin and Haviland presented a four moment solution for hard sphere molecules and a six moment solution for Maxwellian molecules. All of the foregoing analy$\operatorname{ses}^{6-10}$ were developed only for complete thermal accommodation at the surfaces $\left(\alpha_{H}=\alpha_{C}=1\right)$. In order to compare the data to the analytical results here the moment method $^{8}$ is extended to include incomplete thermal accommodation at both the hot and cold surfaces $\left(\alpha_{H} \neq 1\right.$ and $\left.\alpha_{C} \neq 1\right)$. The details of the calculations are briefly outlined in the Appendix. Incomplete thermal accommodation is included only in the Liu-Lees solution ${ }^{8}$ (i.e., four moments, Maxwellian molecules), because, as was shown by Lavin and Haviland, the density distributions given by the four or six moment solutions agree very closely (at least for $\alpha_{H}=$ $\alpha_{C}=1$ ) for hard sphere and Maxwellian molecules.

Comparisons between the data and the results of Liu and Lees' four moment method are shown in Fig. 3. In computing the analytical results the thermal accommodation coefficients measured in the free molecule regime were used with the assumption that their value remained constant over the entire pressure range.

As can be seen from Fig. 3 there is reasonable agreement between the analytical results and the data, the agreement being better at decreasing Knudsen numbers. The data agree well with the analytical results near the cold plate where the local Lnudsen number is less than the indicated value $\left(\mathrm{Kn}_{0}\right)$. Near the hot plate, where the local Knudsen number is higher than the one at the centerplane, the difference between the experimental and analytical results is about $7 \%$.

The question may now be raised whether this difference is caused by end effects which, as pointed out previously, would be more pronounced near the hot plate. According to the data of Teagan and Springer, ${ }^{1}$ under the conditions of our present experiments, end effects would not penetrate to a distance beyond about two to three mean free paths from the ends of the plates. Thus, in our 
experiments the data should not be influenced by end effects near the hot plate. This fact is further supported by the following observation: The local mean free path near the cold plate for $\mathrm{Kn}_{0}=0.203$ is about the same as the local mean free path near the hot plate for $\mathrm{Kn}_{0}=0.053$. However, there is excellent agreement between theory and data near the cold plate at $\mathrm{Kn}_{0}=0.203$, indicating that the difference between the analytical results and the data cannot be readily attributed to end effects.

The results for nitrogen are shown in Fig. 4. These density profiles are very similar to those for helium. In Fig. 4, the density distributions according to the continuum theory $\left(\mathrm{Kn}_{0} \rightarrow 0\right)$ are also shown. Unfortunately, there were no other analytical results available with which to compare these measurements. However, the data for nitrogen (and also for helium) are presented in sufficient detail to be useful in comparisons with forthcoming analytical and numerical results.

\section{ACKNOWLEDGMENTS}

The authors wish to thank Mr. H. Ehya for his help in the experiments.

This work was supported by the United States Atomic Energy Commission.

\section{APPENDIX}

In the following, the four moment method of solution of Liu and Lees ${ }^{8}$ is outlined including incomplete thermal accommodation at the hot and cold surfaces. Liu and Lees proposed to evaluate the conductive heat transfer between two parallel plates by dividing the gas molecules (in velocity space) into two groups. Each of these groups is characterized by a Maxwellian distribution function

$$
f_{1,2}=\frac{n_{1,2}}{\left(2 \pi R T_{1,2}\right)^{3 / 2}} \exp \left(-\frac{\xi^{2}}{2 R T_{1,2}}\right)
$$

where $R$ is the gas constant, $\xi$ is the molecular velocity, and $n$ and $T$ are as yet unknown space dependent constants. The subscripts 1 and 2 refer to the two groups of particles for which $\xi_{x}>0$ and $\xi_{x}<0$. Once the four unknowns $T_{1}, T_{2}, n_{1}$, and $n_{2}$ are known, the density at any point can be readily calculated from the expression ${ }^{8}$

$$
\rho=m n=\int m f d \xi=m \frac{n_{1}+n_{2}}{2} .
$$

The four constants are evaluated by taking moments of the Maxwell integral equation of transfer

$$
\frac{d}{d x}\left(\int f \xi_{x} Q d \xi\right)=\Delta Q,
$$

where $Q$ is any arbitrary function of velocity and $\Delta Q$ represents the moments of the collision integral. By setting $Q=m, m \xi_{x}, \frac{1}{2} m \xi^{2}$, and $\frac{1}{2} m \xi_{x} \xi^{2}$ the following four equations are generated ${ }^{8}$ :

$$
\begin{gathered}
n_{1} T_{1}-n_{2} T_{2}=0, \\
n_{1} T_{1}+n_{2} T_{2}=B_{1}, \\
n_{2} T_{2}^{3 / 2}-n_{1} T_{1}^{3 / 2}=B_{2} B_{3}, \\
\frac{d}{d x}\left(n_{1} T_{1}^{2}+n_{2} T_{2}^{2}\right)+\frac{4}{15} \frac{L n_{c}}{\lambda_{0} n_{0}} B_{2} B_{3}\left(n_{1}+n_{2}\right)=0 .
\end{gathered}
$$

$\lambda_{0}$ and $n_{0}$ are the mean free path and number density at $x=0$, and $n_{c}$ is the number density at the cold wall. The integration constants $B_{1}, B_{2}$, and $B_{3}$ must be evaluated from Eq. (A4) and the boundary conditions. Liu and Lees performed the calculations assuming complete thermal accommodation at the surfaces. Here, we obtain solutions to Eqs. (A4) for the following boundary conditions ${ }^{8,11}$ :

$$
\begin{array}{ll}
x=\frac{L}{2}, & \alpha_{H}=\frac{T_{1}-T_{2}}{T_{1}-T_{H}}, \\
x=-\frac{L}{2}, & \alpha_{C}=\frac{T_{2}-T_{1}}{T_{2}-T_{C}}, \\
x=0, & n_{0}=\frac{\rho_{0}}{m}=\frac{n_{1}+n_{2}}{2} .
\end{array}
$$

Equations (A4) and (A5) were solved numerically for $n_{1}, n_{2}, T_{1}$, and $T_{2}$ for the experimentally determined values of $\alpha_{H}, \alpha_{C}, T_{H}, T_{C}$, and $\rho_{0}$. The resulting density values [Eq. (A2)] are given in Fig. 3 .

${ }^{1}$ W. P. Teagan and G. S. Springer, Phys. Fluids 11, 497 (1968).

${ }_{2}^{2}$ W. P. Teagan and G. S. Springer, Rev. Sci. Instr. 38, 335 (1967).

${ }^{3}$ E. H. Kennard, Kinetic Theory of Gases (McGraw-Hill, New York, 1938), p. 315.

4 E. R. G. Eckert and W. O. Carlson, Intern. J. Heat Mass Transfer 2, 106 (1961).

5 G. S. Springer and W. H. Lipkea, Intern. J. Heat Mass Transfer 11, 1341 (1968).

${ }^{B} \mathrm{C}$. Cercignani and G. Tironi, in Rarefied Gas Dynamics, edited by C. L. Brundin (Academic, New York, 1966), Vol. I, p. 441 .

7 D. R. Willis, in Rarefied Gas Dynamics, edited by J. A. Lauermann (Academic, New York, 1963), Vol. I, p. 209.

${ }^{8}$ C. Y. Liu and L. Lees, in Rarefied Gas Dynamics, edited by L. Talbot (Academic, New York, 1961), p. 391 .

${ }^{9}$ M. L. Lavin and J. K. Haviland, Phys. Fluids 5, 274 (1962).

${ }_{11}^{10}$ L. Lees, J. Soc. Ind. Appl. Math. 13, 278 (1965).

11 F. C. Hurlbut, Phys. Fluids 7, 904 (1964). 